University of Wollongong

Research Online

Faculty of Engineering and Information

Faculty of Engineering and Information

Sciences - Papers: Part A

Sciences

$1-1-2012$

\title{
Nonlinear optical response of graphene superlattice in terahertz frequency regime
}

Yee Sin Ang

University of Wollongong, ysa190@uowmail.edu.au

Chao Zhang

University of Wollongong, czhang@uow.edu.au

Follow this and additional works at: https://ro.uow.edu.au/eispapers

Part of the Engineering Commons, and the Science and Technology Studies Commons

Research Online is the open access institutional repository for the University of Wollongong. For further information contact the UOW Library: research-pubs@uow.edu.au 


\title{
Nonlinear optical response of graphene superlattice in terahertz frequency regime
}

\author{
Abstract \\ We investigate the nonlinear optical response of a Kronig-Penney type graphene superlattice in which the \\ Fermi velocity of the massless Dirac quasiparticle anisotropically depends on the direction of \\ propagation. Such velocity anisotropy results in enhanced nonlinear optical response in terahertz \\ frequency regime.

\section{Keywords} \\ frequency, optical, nonlinear, regime, terahertz, superlattice, graphene, response

\section{Disciplines} \\ Engineering | Science and Technology Studies

\section{Publication Details} \\ Ang, Y. \& Zhang, C. (2012). Nonlinear optical response of graphene superlattice in terahertz frequency \\ regime. Laser and Tera-Hertz Science and Technology, LTST 2012 (pp. STh4A.22-1-STh4A.22-2). United \\ States: Optical Society of America.
}




\title{
Nonlinear Optical Response of Graphene Superlattice in Terahertz Frequency Regime
}

\author{
Yee Sin Ang and Chao Zhang \\ School of Physics, University of Wollongong, New South Wales, 2522 Australia \\ czhang@uow.edu.au
}

\begin{abstract}
We investigate the nonlinear optical response of a Kronig-Penney type graphene superlattice in which the Fermi velocity of the massless Dirac quasiparticle anisotropically depends on the direction of propagation. Such velocity anisotropy results in enhanced nonlinear optical response in terahertz frequency regime.
\end{abstract}

\section{Introduction}

Graphene is a two-dimensional nanostructure made up of entirely carbon atoms. Many interesting physical properties of graphene lie on its low energy electrons residing at the corner of the Brillouine zone [1-3]. These electrons behave like massless ultra-relativistic fermion and follows linear energy dispersion. Graphene and bilayer graphene exhibits exceptionally strong nonlinear optical response in the useful terahertz (THz) frequency regime, requiring only a very modest electric field strength of $\mathrm{E} \approx 1000 \mathrm{~V} / \mathrm{cm}$ to achieve observable nonlinear optical response $[4,5]$. The low energy graphene band structure is in the form of a 'Dirac cone' with Fermi velocity of $v_{F} \approx$ $10^{6} \mathrm{~m} / \mathrm{s}$ and is isotropic in all direction. When a periodic Kronig-Penney potential is applied, the graphene Fermi velocity perpendicular to the periodicity of the external potential is renormalized $[6,7]$. The quasiparticle in a Kronig-Penney type (KP) graphene superlattice therefore becomes an anisotropic Dirac fermion, mimicking a peculiar massless ultra-relativistic fermion propagating in anisotropic space-time.

In this work, we study the terahertz $(\mathrm{THz})$ regime nonlinear optical response of graphene superlattice structure in the presence of Dirac fermion anisotropy. We found that the nonlinear optical response is enhanced by the Dirac fermion anisotropy. Since the degree of Dirac fermion anisotropy can be continuously tuned by varying the KP potential profile, graphene superlattice can potentially be utilized as a $\mathrm{THz}$ detector/emitter with great practicality and flexibility.

\section{Models}

The low energy effective Hamiltonian of the anisotropic Dirac fermions in KP graphene superlattice can be modeled by

$$
H=v_{F}\left[\begin{array}{cc}
0 & p_{x}-i \lambda p_{y} \\
p_{x}+i \lambda p_{y} & 0
\end{array}\right]
$$

where $v_{F} \approx 10^{6} \mathrm{~m} / \mathrm{s}$ and $\lambda$ is a dimensionless anisotropy parameter and can be continuously tuned in the range of $0<\lambda<1$ by adjusting the KP external potential. The Dirac fermion travels anisotropically with $v_{F}$ in the direction along the periodicity of the KP potential (x-direction) and with a reduced velocity of $\lambda v_{F}$ in the direction perpendicular to the KP potential periodicity (y-direction). When an external AC electric field $\mathbf{E}(\mathrm{t})=\mathbf{E} \mathrm{e}^{\mathrm{i} \omega t}$ is applied along the $\mathrm{x}$-direction, the quasiparticle can be coupled to the photon via the substitution $\boldsymbol{p} \rightarrow \boldsymbol{p}-\boldsymbol{e A}$ and the quasiparticle wavefunction can be expressed as an infinite sum

$$
\psi=\sum_{n=0}^{\infty} \phi_{n}(\vec{p}) e^{i(n \omega-\varepsilon / \hbar) t}=\sum_{n=0}^{\infty}\left[\begin{array}{l}
a_{n}(\vec{p}) \\
b_{n}(\vec{p})
\end{array}\right] e^{i(n \omega-\varepsilon / \hbar) t}
$$

where each $\mathrm{n}^{\text {th }}$ term represents electronic states coupled to $\mathrm{n}$-photon. The optical current can then be obtained from the current density equation

$$
\mathbf{J}_{n}^{v}=\frac{e}{4 \pi^{2}} \int d \mathbf{p} \phi_{n}^{+} v_{\nu} \phi_{n} N(\varepsilon)
$$

where the velocity operator is given as $v_{v}=\partial H / \partial p_{v}$ with $v=(x, y)$, and $N(\varepsilon)=f(-\varepsilon)-f(\varepsilon)$ is a thermal factor describing the availability of the valence band charge carrier and the thermal occupancy of the conduction band. 


\section{Results and Discussions}

The temperature dependence of the third order nonlinear optical response is plotted in Fig. 1 at $1 \mathrm{THz}$ and electric field strength $1000 \mathrm{~V} / \mathrm{cm}$. The third order response is made up of two distinct components: (i) $\sigma_{3}(\omega)$, singlefrequency term which corresponds to the absorption of two photons and the simultaneous emission of one photon; and (ii) $\sigma_{3}(3 \omega)$, triple-frequency term which corresponds to the simultaneous absorption of three photons. The nonlinear response is reduced at higher temperature since the valance band is thermally evacuated while the conduction band becomes thermally occupied. In comparison to the isotropic case $(\lambda=1)$, the anisotropic Dirac fermions $(\lambda=0.25)$ exhibit a stronger nonlinear optical absorption. The nonlinear optical response is found to be enhanced by a factor of $1 / \lambda$. This surprising anisotropy-induced optical response enhancement can be understood by the fact that as the Fermi velocity in y-direction is reduced to $\lambda v_{F}$, the smaller slope along the y-direction of the band structure results in larger y-component momentum $p_{y}$ across a constant energy slice in comparison to the isotropic case. The overall larger momentum of the charge carrier therefore leads to the enhanced optical response.

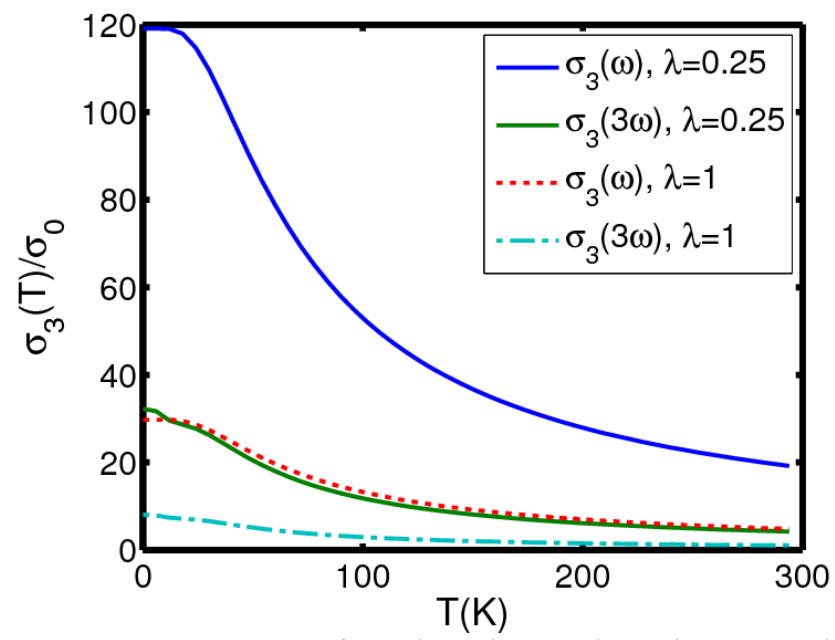

Fig. 1 - Nonlinear optical conductivities of graphene $\lambda=1$ and graphene superlattice with $\lambda=0.25$.

\section{Conclusions}

In conclusion, the nonlinear optical response of graphene superlattice in terahertz frequency regime was found to be enhanced due to the presence of anisotropic Dirac fermions. The anisotropy can be continuously tuned by adjusting the Kronig-Penney potential profile. This suggests that graphene superlattice can potentially be utilized as a convenient tunable $\mathrm{THz}$ waves detector/emitter.

\section{References}

[1] A. K. Geim and K. S. Novoselov, Nature Mater. 6, 183 - 191 (2007).

[2] A. H. Castro Neto, F. Guinea and N. M. R. Peres, Rev. Mod. Phys. 81, 109 - 162 (2009).

[3] C. Zhang, L. Chen, and Z. S. Ma,, Phys. Rev. B. 77, 241402(R) (2008)

[4] Junfeng Liu, A. R. Wright, Chao Zhang, Zhongshui. Ma, Appl. Phys. Lett. 93, 041106 (2008)

[5] Y. S. Ang, S. Sultan and C. Zhang, Appl. Phys. Lett. 97, 243110 (2010).

[6] C. H. Park, L. Yang, Y. W. Son, M. L. Cohen and S. G. Louie, Nature Phys. 4, 213 - 217 (2008).

[7] C. H. Park, L. Yang, Y. W. Son, M. L. Cohen and S. G. Louie, Phys. Rev. Lett. 101, 126804 (2008). 